\title{
Quantum theory of electromagnetic fields in a cosmological quantum spacetime
}

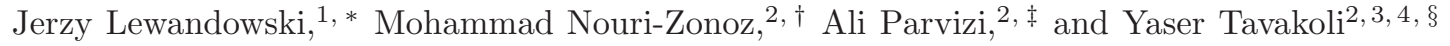 \\ ${ }^{1}$ Faculty of Physics, University of Warsaw, Pasteura 5, 02-093 Warsaw, Poland \\ ${ }^{2}$ Department of Physics, University of Tehran, 14395-547 Tehran, Iran \\ ${ }^{3}$ School of Engineering Science, College of Engineering, University of Tehran, 11155-4563 Tehran, Iran \\ ${ }^{4}$ School of Physics, Institute for Research in Fundamental Sciences (IPM), 19395-5531 Tehran, Iran
}

(Dated: November 7, 2017)

\begin{abstract}
The theory of quantum fields propagating on an isotropic cosmological quantum spacetime is reexamined by generalizing the scalar test field to an electromagnetic (EM) vector field. For any given polarization of the EM field on the classical background, the Hamiltonian can be written in the form of the Hamiltonian of a set of decoupled harmonic oscillators, each corresponding to a single mode of the field. In transition from the classical to quantum spacetime background, following the technical procedure given by Ashtekar et al. [Phys. Rev. D 79, 064030 (2009)], a quantum theory of the test EM field on an effective (dressed) spacetime emerges. The nature of this emerging dressed geometry is independent of the chosen polarization, but it may depend on the energy of the corresponding field mode. Specifically, when the backreaction of the field on the quantum geometry is negligible (i.e., a test field approximation is assumed), all field modes probe the same effective background independent of the mode's energy. However, when the backreaction of the field modes on the quantum geometry is significant, by employing a Born-Oppenheimer approximation, it is shown that a rainbow (i.e., a mode-dependent) metric emerges. The emergence of this mode-dependent background in the Planck regime may have a significant effect on the creation of quantum particles. The production amount on the dressed background is computed and is compared with the familiar results on the classical geometry.
\end{abstract}

PACS numbers: 04.60.-m, 04.60.Pp, 98.80.Qc

\section{INTRODUCTION}

It is argued that the theory of quantum fields in the Planck regime requires quantization of the given background spacetime [1-3]. It is then that one could study the dynamics of quantum fields by analyzing the field modes propagating on these quantum geometries. This issue has already been studied when a test scalar field propagates on a quantum isotropic [4] and the simplest anisotropic [5] background spacetime. An extension of those models was also provided in Ref. [6].

In the loop quantum cosmological background [7, 8], one regards the background matter source $T$ (i.e., given by a scalar field [9] or a dust field [10]) as a global relational time variable with respect to which physical observables evolve. This simplifies the task of solving the constraints and constructing the physical sector of the theory and enables one to introduce convenient Dirac observables for the quantum theory. Then, on the full phase space of the loop quantum cosmology (LQC), the background geometry is described by a state $\Psi_{o}$ that evolves, with respect to $T$, via a Hamiltonian $\hat{H}_{o}$. The state $\Psi_{o}$ undergoes a quantum bounce at some time $T=T_{\mathrm{B}}$. The quantum state of inhomogeneous fields $\varphi$, propagating on this quantum background, is denoted by $\psi$, which pos-

\footnotetext{
*Electronic address: jerzy.lewandowski@fuw.edu.pl

${ }^{\dagger}$ Electronic address: nouri@ut.ac.ir

${ }^{\ddagger}$ Electronic address: a.parvizi@ut.ac.ir

$\S$ Electronic address: yaser.tavakoli@ut.ac.ir
}

sesses a natural initial condition at $T_{\mathrm{B}}$.

When the massless scalar inhomogeneities are regarded as perturbations, the backreaction between fields and the geometry can be discarded, and thus their state $\psi$ can be chosen such that their energy density is small at $T_{\mathrm{B}}$. If this situation continues to sustain at later times, then $\Psi_{o} \otimes \psi$ would be a self-consistent solution to the total Hamiltonian constraint of the gravity-field system, for all times. In this case, each massless mode of the scalar field on the quantum background probes an effective dressed geometry that is independent of the wave number of the mode. Moreover, the emergent geometry has the same isotropy of the original background metric. For massive modes, however, quantum effects may give rise to a small deviation for the isotropy of the background [11]. Furthermore, in some situations, a rainbow metric may emerge, a metric that has a dependence on the energy of the field modes propagating on it [12, 13]. Including the backreaction, as $\Psi_{o} \otimes \psi+\delta \Psi$, can have a significant effect on the total state of the geometry-field system, which may also lead to violation of the local Lorentz symmetry [5]. On the other hand, such a quantum geometry provides only probability amplitudes for various homogeneous metrics, and thus we no longer have a sharply defined, proper, or conformal time variable [9]. This issue was resolved by studying the dynamics of inhomogeneous perturbations on a quantum Friedmann-LemaîtreRobertson-Walker (FLRW) spacetime, by deparametrizing the Hamiltonian constraint in the background, homogeneous sector [14].

After constructing quantum electrodynamics on the flat spacetime, it is of interest to generalize the formu- 
lation to the general curved spacetimes. Illness of the notion of a particle and lack of Poincaré symmetry in general spacetime makes it difficult to study quantum theory of fields in spacetimes without preferred symmetries associated with a killing vector. Cosmological backgrounds are among the first targets in studying the quantization of electromagnetic (EM) fields in curved backgrounds; any observation made in cosmological scales is related to the analyses of the EM fields, from classical to quantum phenomenons. We are especially interested in investigating the behavior of quantum EM fields on cosmological quantum backgrounds. There are many quantum gravitational phenomena occurring in the cosmos, and EM fields originating from them carry valuable information about quantum gravitational effects taking place in that part of the Universe. There are significant studies proposing the violation of the Lorentz symmetry due to quantum gravity effects, such as spectral lag in gammaray bursts and observations indicating that the speed of light in vacuum depends on the energy of the photons [15 17] (for further phenomenological aspects of quantum gravity, see Refs. [18 22]). It was also shown that quantum gravity effects may also imprint signatures on the cosmic microwave background observations [23]. All of these phenomena convince us to study the quantum theory of EM fields not only on the classical cosmological spacetime but also on the quantum cosmological backgrounds.

The paper is organized as follows. In Sec. III. we study the Hamiltonian formalism of a quantum EM vector field on a flat FLRW background. In Sec. III, we will present a quantum background on which the EM perturbations can propagate. We will discuss two particular situations: the case in which the backreaction can be neglected and the case in which the backreaction effect is significant in the quantum gravity regime. We will show that, in the presence of backreaction, a deviation from the local Lorentz symmetry emerges. In Sec. [V we will discuss phenomenological aspects of the Lorentz symmetry breaking on the creation of particles in quantum spacetime and then compare our results with the well-known studies of quantized fields on a classical isotropic background. Finally, in Sec. VI comes the conclusion and discussion of our work.

\section{QUANTUM THEORY OF RADIATION FIELD IN A CLASSICAL SPACETIME}

In this section, we will study the Hamiltonian formulation of an EM field propagating in a flat FLRW background spacetime.

\section{A. EM field equation in flat FLRW spacetime: Radiation gauge}

We consider a four-dimensional curved background spacetime, which is equipped with the coordinates $\left(x_{0}, \mathbf{x}\right)$, where the spatial coordinates $\mathbf{x} \in(0, \ell)$ on a torus $\mathbb{T}^{3}$ and $x_{0} \in \mathbb{R}$ is a generic time coordinate. Let us consider a free EM field on this background spacetime, the Lagrangian density (in vacuum) of which is given by

$$
\mathcal{L}_{\mathrm{EM}}=-\frac{1}{4} \sqrt{-g} F_{a b} F^{a b},
$$

where $F_{a b}$ is the EM field, being a covariant antisymmetric tensor of rank 2, which can be defined in terms of the EM potential $A_{a}$ by

$$
F_{a b}=\partial_{a} A_{b}-\partial_{b} A_{a}
$$

and $F^{a b}=g^{a c} F_{c d} g^{d b}$. The Maxwell equations can be written in terms of the 4-potential $A_{a}$, as [24]

$$
\square A^{a}-A_{b}^{b ; a}=0,
$$

where $\square:=g^{a b} \nabla_{a} \nabla_{b}$. Employing the Lorenz gauge in curved spacetime, $A_{; a}^{a}=0$, it reduces to

$$
\square A^{a}-\mathcal{R}^{a}{ }_{b} A^{b}=0,
$$

with $\mathcal{R}_{a b}$ being the Ricci tensor. Note that Eq. (2.4) has the same form of the wave equation as in flat spacetime, except that the derivatives are replaced by covariant derivatives and there is an additional term proportional to the curvature.

Henceforth, we will study the quantum theory of EM fields propagating on a homogeneous background provided by a flat FLRW model:

$$
g_{a b} d x^{a} d x^{b}=-N_{x_{0}}^{2}\left(x_{0}\right) d x_{0}^{2}+a^{2}\left(x_{0}\right) d \mathbf{x}^{2} .
$$

By writing the d'Alembertian in Eq. (2.4) in terms of the connection coefficients, we have

$$
\begin{aligned}
A_{; ;}^{a ; b}=g^{b c}[ & A^{a}{ }_{, b, c}+\Gamma_{d b, c}^{a} A^{d}+2 \Gamma_{d c}^{a} A^{d}{ }_{, b} \\
& \left.+\Gamma_{b d}^{a} \Gamma_{c e}^{d} A^{e}-\Gamma_{b c}^{d} A^{a}{ }_{, d}-\Gamma_{b c}^{d} \Gamma_{e d}^{a} A^{e}\right] .
\end{aligned}
$$

Computation of the connection coefficients of the metric (2.5) in rectangular coordinates (see Appendix A) shows that there are no terms involving first-order spatial derivatives of the components of the EM potential in the wave equation (2.4). According to the remark mentioned and using the radiation gauge (in which we have $A^{0}=0$ ), the field equation for each spatial component of the vector potential $\mathbf{A}\left(x_{0}, \mathbf{x}\right)$ lines up like

$$
\Delta^{(3)} A^{i}-\frac{a^{2}}{N^{2}} \ddot{A}^{i}+\frac{a^{2}}{N^{2}}\left[\frac{\dot{N}}{N}-5 \frac{\dot{a}}{a}\right] \dot{A}^{i}=0,
$$

where $\Delta^{(3)}$ is the Laplacian associated with the spatial Cartesian coordinates, $i=1,2,3$, and a dot denotes a 
partial derivative with respect to $x_{0}$. Now we can separate $\mathbf{A}\left(x_{0}, \mathbf{x}\right)$ as

$$
\mathbf{A}\left(x_{0}, \mathbf{x}\right)=\underline{\mathbf{A}}\left(x_{0}\right) u(\mathbf{x})
$$

with $u(\mathbf{x})$ a solution of

$$
\Delta^{(3)} u(\mathbf{x})=-k^{2} u(\mathbf{x})
$$

In other words, $u(\mathbf{x})$ is the eigenfunction of $\Delta^{(3)}$ operator with a plane wave solution, i.e., the spatial part of the EM vector potential $\mathbf{A}$ could be expanded in terms of plane waves.

Performing the Legendre transformation, one gets the electric field $E^{i}$ as the canonically conjugate momentum for the corresponding component of the vector potential $A_{i}$, on a $x_{0}=$ const. slice [see Eq. (B4)]. Then, for the pair $\left(A_{i}, E^{i}\right)$, the classical solutions of the equation of motion (2.4) can be expanded in Fourier modes as

$$
\begin{aligned}
& \mathbf{A}\left(x_{0}, \mathbf{x}\right)=\frac{1}{(2 \pi)^{3 / 2}} \sum_{\mathbf{k} \in \mathcal{L}} \sum_{r}^{2} \mathbf{A}_{\mathbf{k}}^{r}\left(x_{0}\right) e^{i \mathbf{k} \cdot \mathbf{x}} \\
& \boldsymbol{\pi}\left(x_{0}, \mathbf{x}\right)=\frac{1}{(2 \pi)^{3 / 2}} \sum_{\mathbf{k} \in \mathcal{L}} \sum_{r}^{2} \boldsymbol{\pi}_{\mathbf{k}}^{r}\left(x_{0}\right) e^{i \mathbf{k} \cdot \mathbf{x}} .
\end{aligned}
$$

The wave vector $\mathbf{k} \in(2 \pi \mathbb{Z} / \ell)^{3}$ spans a three-dimensional lattice $\mathcal{L}$, with $\mathbb{Z}$ being the set of integers [5] (see Appendix B] . Notice that, since the fields are purely inhomogeneous, the zero $\mathbf{k}$ is excluded.

For each mode $\mathbf{k}$, the Fourier coefficients $\mathbf{A}_{\mathbf{k}}^{r}$ and $\boldsymbol{\pi}_{\mathbf{k}}^{r}$ must evidently be vectors,

$$
\mathbf{A}_{\mathbf{k}}^{r}=\mathrm{A}_{\mathbf{k}}^{r} \epsilon_{\mathbf{k}}^{r}, \quad \text { or } \quad \pi_{\mathbf{k}}^{r}=\pi_{\mathbf{k}}^{r} \epsilon_{\mathbf{k}}^{r},
$$

which should satisfy the given gauge conditions. Here, $\boldsymbol{\epsilon}_{\mathbf{k}}^{r}$ are the so-called polarization vectors [25]. From the radiation gauge condition $\nabla \cdot \mathbf{A}=0$, we have that

$$
\mathbf{A}_{\mathbf{k}}^{r} \cdot \mathbf{k}=0, \quad \text { or } \quad \boldsymbol{\epsilon}_{\mathbf{k}}^{r} \cdot \mathbf{k}=0 .
$$

So, for a given direction of propagation $\mathbf{k} /|\mathbf{k}|$, the polarization vectors are transverse. Moreover, they may also be chosen to be orthonormal; $\boldsymbol{\epsilon}_{\mathbf{k}}^{r} \cdot \boldsymbol{\epsilon}_{\mathbf{k}}^{r^{\prime}}=\delta_{r r^{\prime}}$. Therefore, for each Fourier coefficient, we have two polarization vectors; i.e., $r=1,2$.

\section{B. Hamiltonian of the EM field}

From the Lagrangian (2.1), we can write the Hamiltonian form of the EM field propagating on the classical FLRW spacetime:

$$
\mathcal{H}_{\mathrm{EM}}=\frac{N_{x_{0}}}{2 a^{3}}\left[\boldsymbol{\pi} \cdot \boldsymbol{\pi}+a^{6} \sum_{i<j} F_{i j} F^{i j}\right]
$$

In terms of the conjugated pairs $\left(A_{i}, \pi^{i}\right)$, defined in Eq. (B4), the total Hamiltonian of the EM field is obtained as ${ }^{1}$

$$
\begin{aligned}
H_{\mathrm{EM}} & =\int d^{3} x \mathcal{H}_{\mathrm{EM}} \\
& =\frac{N_{x_{0}}}{2 a^{3}} \int d^{3} x\left[\boldsymbol{\pi} \cdot \boldsymbol{\pi}+a^{6} \partial_{i} \mathbf{A} \cdot \partial^{i} \mathbf{A}\right] .
\end{aligned}
$$

Substituting for $A_{i}$ and $\pi^{i}$ from (2.10) and (2.11), we find $^{2}$

$$
H_{\mathrm{EM}}=\frac{N_{x_{0}}}{2 a^{3}} \sum_{\mathbf{k}} \sum_{r}^{2}\left[\left(\pi_{\mathbf{k}}^{r}\right)^{*} \pi_{\mathbf{k}}^{r}+k^{2} a^{4}\left(\mathrm{~A}_{\mathbf{k}}^{r}\right)^{*} \mathrm{~A}_{\mathbf{k}}^{r}\right] .
$$

The reality condition for the EM field $\mathbf{A}\left(x_{0}, \mathbf{x}\right)$ implies that not all modes $\mathrm{A}_{\mathbf{k}}^{r}\left(x_{0}\right)$ of the field are independent ${ }^{3}$; to understand this better, let us decompose a field mode $\mathrm{A}_{\mathbf{k}}^{r}\left(x_{0}\right)$ and its momentum $\pi_{\mathbf{k}}^{r}\left(x_{0}\right)$ as

$$
\begin{aligned}
\mathrm{A}_{\mathbf{k}}^{r} & :=\frac{1}{\sqrt{2}}\left(\mathrm{~A}_{\mathbf{k}}^{r(1)}+i \mathrm{~A}_{\mathbf{k}}^{r(2)}\right), \\
\pi_{\mathbf{k}}^{r} & :=\frac{1}{\sqrt{2}}\left(\pi_{\mathbf{k}}^{r(1)}+i \pi_{\mathbf{k}}^{r(2)}\right) .
\end{aligned}
$$

Then, from the conditions $\left(\mathrm{A}_{-\mathbf{k}}^{r}\right)^{*}=\mathrm{A}_{\mathbf{k}}^{r}$ and $\left(\pi_{-\mathbf{k}}^{r}\right)^{*}=$ $\pi_{\mathbf{k}}^{r}$, we have that

$$
\begin{array}{ll}
\mathrm{A}_{-\mathbf{k}}^{r(1)}=\mathrm{A}_{\mathbf{k}}^{r(1)}, & \mathrm{A}_{-\mathbf{k}}^{r(2)}=-\mathrm{A}_{\mathbf{k}}^{r(2)}, \\
\pi_{-\mathbf{k}}^{r(1)}=\pi_{\mathbf{k}}^{r(1)}, & \pi_{-\mathbf{k}}^{r(2)}=-\pi_{\mathbf{k}}^{r(2)} .
\end{array}
$$

That is, there exist relations between the "positive" ( $\mathbf{k} \in$ $\left.\mathcal{L}_{+}\right)$and "negative" $\left(-\mathbf{k} \in \mathcal{L}_{-}\right)$modes of the field. This enables one to split the lattice $\mathcal{L}$, for each $\mathbf{k}=\left(k_{1}, k_{2}, k_{3}\right)$, into positive and negative sectors [4],

$$
\begin{gathered}
\mathcal{L}_{+}=\left\{\mathbf{k}: k_{3}>0\right\} \cup\left\{\mathbf{k}: k_{3}=0, k_{2}>0\right\} \\
\cup\left\{\mathbf{k}: k_{3}=k_{2}=0, k_{1}>0\right\}
\end{gathered}
$$

and

$$
\begin{aligned}
& \mathcal{L}_{-}=\left\{\mathbf{k}: k_{3}<0\right\} \cup\left\{\mathbf{k}: k_{3}=0, k_{2}<0\right\} \\
& \cup\left\{\mathbf{k}: k_{3}=k_{2}=0, k_{1}<0\right\} \\
&=\left\{\mathbf{k}:-\mathbf{k} \in \mathcal{L}_{+}\right\},
\end{aligned}
$$

respectively. By using this fact, we are also able to decompose any summation over $\mathbf{k} \in \mathcal{L}$ into its positive and negative parts. In particular, by defining new variables $Q_{\mathbf{k}}^{r}$ and $P_{\mathbf{k}}^{r}$

$$
\begin{aligned}
& Q_{\mathbf{k}}^{r}:=\left\{\begin{array}{lll}
\mathrm{A}_{\mathbf{k}}^{r(1)} & \text { for } & \mathbf{k} \in \mathcal{L}_{+} \\
\mathrm{A}_{-\mathbf{k}}^{r(2)} & \text { for } & \mathbf{k} \in \mathcal{L}_{-}
\end{array}\right. \\
& P_{\mathbf{k}}^{r}:=\left\{\begin{array}{lll}
\pi_{\mathbf{k}}^{r(1)} & \text { for } & \mathbf{k} \in \mathcal{L}_{+} \\
\pi_{-\mathbf{k}}^{r(2)} & \text { for } & \mathbf{k} \in \mathcal{L}_{-}
\end{array}\right.
\end{aligned}
$$

\footnotetext{
${ }^{1}$ see Eq. B11 in Appendix B

${ }^{2}$ See also Eqs. B12 - B14 for more details.

3 cf., for example, Refs. [4, 26] for the case of a scalar field.
} 
we can reexpress the Hamiltonian (2.16) as

$$
\begin{aligned}
H_{\mathrm{EM}}\left(x_{0}\right) & =\frac{N_{x_{0}}}{2 a^{3}} \sum_{\mathbf{k} \in \mathcal{L}} \sum_{r}^{2}\left[\left(P_{\mathbf{k}}^{r}\right)^{2}+k^{2} a^{4}\left(Q_{\mathbf{k}}^{r}\right)^{2}\right] \\
& =: \sum_{\mathbf{k} \in \mathcal{L}} \sum_{r}^{2} H_{\mathbf{k}}^{(r)}\left(x_{0}\right) .
\end{aligned}
$$

This equation represents the Hamiltonian of a set of decoupled harmonic oscillators defined by conjugate pairs $\left(Q_{\mathbf{k}}^{r}, P_{\mathbf{k}}^{r}\right)$. Here, $Q_{\mathbf{k}}^{r}$ and $P_{\mathbf{k}}^{r}$ are conjugate variables associated with any $\mathbf{k}$ mode, satisfying the relation $\left\{Q_{\mathbf{k}}^{r}, P_{\mathbf{k}^{\prime}}^{r^{\prime}}\right\}=\delta_{\mathbf{k k}^{\prime}} \delta_{r r^{\prime}}$.

In quantum theory, quantization of a single mode $\mathbf{k}$ (for each polarization $r$ ) of the vector potential, is carried out in the same way as for the quantum harmonic oscillator with the dynamical variables promoted to operators on the Hilbert space $\mathscr{H}_{r, \mathbf{k}}=L^{2}\left(\mathbb{R}, d Q_{\mathbf{k}}^{r}\right)$, as

$$
\hat{Q}_{\mathbf{k}}^{r} \psi\left(Q_{\mathbf{k}}^{r}\right)=Q_{\mathbf{k}}^{r} \psi\left(Q_{\mathbf{k}}^{r}\right)
$$

and

$$
\hat{P}_{\mathbf{k}}^{r} \psi\left(Q_{\mathbf{k}}^{r}\right)=-i \hbar\left(\partial / \partial Q_{\mathbf{k}}^{r}\right) \psi\left(Q_{\mathbf{k}}^{r}\right)
$$

Then, the time evolution of any state $\psi\left(Q_{\mathbf{k}}^{r}\right)$ of the system is generated by the Hamiltonian operator $\hat{H}_{\mathbf{k}}^{(r)}$ via the Schrödinger equation:

$i \hbar \partial_{x_{0}} \psi\left(x_{0}, Q_{\mathbf{k}}^{r}\right)=\frac{N_{x_{0}}}{2 a^{3}}\left[\left(\hat{P}_{\mathbf{k}}^{r}\right)^{2}+k^{2} a^{4}\left(\hat{Q}_{\mathbf{k}}^{r}\right)^{2}\right] \psi\left(x_{0}, Q_{\mathbf{k}}^{r}\right)$.

In the present study, the wave functions of the EM modes evolve with respect to a general time coordinate $x_{0}$ with the lapse $N_{x_{0}}$.

Equation (2.27) shows that, by fixing $N_{x_{0}}=a(\eta)$ (i.e., a conformal time coordinate $x_{0}=\eta$ ), the evolution equation of the quantum EM field on the FLRW background reduces to the Schrödinger equation for the wave function of the same EM field on a flat Minkowski space with the coordinates $\left(\eta, x^{i}\right)$. More precisely, the corresponding (classical) equation of motion for each mode $Q_{\mathrm{k}}^{r}$, for a given Hamiltonian $H_{\mathbf{k}}^{(r)}\left(x_{0}\right)$ in Eq. (2.24), reads

$$
\ddot{Q}_{\mathbf{k}}^{r}+\frac{\dot{m}}{m} \dot{Q}_{\mathbf{k}}^{r}+\omega_{k}^{2}\left(x_{0}\right) Q_{\mathbf{k}}^{r}=0
$$

where we have defined $m\left(x_{0}\right)=a^{3} / N_{x_{0}}$ and $\omega_{k}^{2}\left(x_{0}\right)=$ $N_{x_{0}}^{2} k^{2} / a^{2}$. For $N_{x_{0}}=a^{3}(\tau)$, Eq. (2.28) reduces to

$$
\ddot{Q}_{\mathbf{k}}^{r}+k^{2} a^{4} Q_{\mathbf{k}}^{r}=0
$$

\section{QUANTUM EM FIELD ON QUANTIZED BACKGROUND}

In this section, we briefly review Ref. [10] by considering a FLRW metric (2.5) coupled with an irrotational dust, as a background matter source, and then present the LQC quantization procedure in order to quantize the gravity-matter system.

Let us consider a background gravitational system coupled to an irrotational dust $T$, which is homogeneous on the spatial slices of the background metric (2.5), and its Lagrangian density is given by [10]

$$
\mathcal{L}_{\mathrm{D}}=-\frac{1}{2} \sqrt{-g} M\left(g^{a b} \partial_{a} T \partial_{b} T+1\right),
$$

with $M$ enforcing the gradient of the dust field to be timelike. Then, the corresponding action for the background geometry together with a standard model matter (which denotes the EM field action $S_{\mathrm{EM}}$ for our purpose in this paper) reads

$$
S=\int d^{4} x\left[\frac{\sqrt{-g}}{8 \pi G} \mathcal{R}+\mathcal{L}_{\mathrm{D}}\right]+S_{\mathrm{EM}} .
$$

The total Hamiltonian, including the gravitational and matter sectors is given by

$$
H=\int d^{3} x\left[\mathcal{H}_{\mathrm{gr}}+\mathcal{H}_{\mathrm{D}}+\mathcal{H}_{\mathrm{EM}}\right]
$$

where $\mathcal{H}_{\mathrm{gr}}, \mathcal{H}_{\mathrm{D}}$, and $\mathcal{H}_{\mathrm{EM}}$ are, respectively, the Hamiltonian density of the gravitational, dust, and EM field sectors. The dust contribution $\mathcal{H}_{\mathrm{D}}$ to the total Hamiltonian constraint is given by

$$
\mathcal{H}_{\mathrm{D}}=N_{x_{0}} \sqrt{p_{T}^{2}+q^{a b} C_{a}^{D} C_{b}^{D}},
$$

where $p_{T}$ is the momentum conjugate of $T$, given by

$$
p_{T}=\sqrt{q} \frac{M}{N_{x_{0}}}\left(\dot{T}+N^{a} \partial_{a} T\right) .
$$

Moreover, $C_{a}^{D}$ is the spatial diffeomorphism constraint:

$$
C_{a}^{D}=-p_{T} \partial_{a} T
$$

For the homogeneous spatial slices, here, we have $\partial_{i} T=0$, so from Eq. (3.5), we get $p_{T}=\sqrt{q} M \dot{T} / N_{x_{0}}$ and $C_{a}^{D}=0$. Using this condition in the dust Hamiltonian density (3.4), together by imposing the canonical time gauge fixing condition (i.e., $N_{x_{0}}=1$ so that $\dot{T}=1$ and $x_{0}=t=T$, which is an obvious choice for the parametrized particle and scalar field), we can write the total Hamiltonian constraint, including the background gravity-dust system together with the EM field, on the full phase space:

$$
\begin{aligned}
H & =\int d^{3} x\left[p_{T}+\mathcal{H}_{\mathrm{gr}}\right]+H_{\mathrm{EM}} \\
& =\ell^{3}\left[p_{T}+\mathcal{H}_{\mathrm{gr}}\right]+H_{\mathrm{EM}} \approx 0 .
\end{aligned}
$$

At this step, the physical Hamiltonian density can be defined as

$$
\tilde{\mathcal{H}}:=-p_{T}=\mathcal{H}_{\mathrm{gr}}+\frac{H_{\mathrm{EM}}}{\ell^{3}}
$$


For the FLRW metric (2.5), the EM Hamiltonian $H_{\mathrm{EM}}$ is given by Eq. (2.24), and the gravitational Hamiltonian reads [, 27]

$$
H_{\mathrm{gr}}=\int d^{3} x \mathcal{H}_{\mathrm{gr}}=\frac{3 \pi G}{2 \alpha} b^{2}|v|,
$$

where $b / \gamma=\dot{a} / a$ is the Hubble parameter with $\gamma$ being the so-called Barbero-Immirzi parameter of LQG. Moreover, $\alpha=2 \pi \gamma \sqrt{\Delta} \ell_{\mathrm{Pl}}^{2} \approx 1.35 \ell_{\mathrm{Pl}}^{3}$ (where $\Delta$ is the so-called "area gap" given by $\Delta=3 \sqrt{3} \pi \gamma \ell_{\mathrm{Pl}}^{2}$ ), and $v=\ell^{3} a^{3} / \alpha$ is the oriented volume with $b$ being its conjugate momentum satisfying $\{v, b\}=2$.

Following the Dirac quantization scheme for the constrained systems, a total kinematical Hilbert space for the above gravity-matter system can be defined as $\mathscr{H}_{\text {kin }}=$ $\mathscr{H}_{\mathrm{gr}} \otimes \mathscr{H}_{T} \otimes \mathscr{H}_{\mathrm{EM}}$, where the matter sectors are quantized according to the Schrödinger picture with the Hilbert spaces $\mathscr{H}_{T}=L^{2}(\mathbb{R}, d T)$ and $\mathscr{H}_{r, \mathbf{k}}=L^{2}\left(\mathbb{R}, d Q_{\mathbf{k}}^{r}\right.$ ) (for each mode), and gravity is quantized due to LQC with $\mathscr{H}_{\mathrm{gr}}=L^{2}\left(\overline{\mathbb{R}}, d \mu_{\mathrm{Bohr}}\right)$ (in which $\overline{\mathbb{R}}$ is the Bohr compactification of the real line and $d \mu_{\mathrm{Bohr}}$ is the Haar measure on it [7]). Now, from Eq. (3.8), the corresponding quantum operators on $\mathscr{H}_{\text {kin }}$ are those acting on physical states $\Psi(v, Q, T) \in \mathscr{H}_{\text {kin }}$ such that

$$
i \hbar \partial_{T} \Psi(v, Q, T)=\left[\hat{\mathcal{H}}_{\mathrm{gr}}+\hat{H}_{\mathrm{EM}} / \ell^{3}\right] \Psi(v, Q, T) .
$$

Here, $v$ is the eigenstate of the volume operator of the quantum background geometry,

$$
\hat{v}|v\rangle=v|v\rangle \quad \text { and } \quad \hat{\mathcal{N}}|v\rangle=|v+1\rangle,
$$

where $\hat{\mathcal{N}} \equiv \widehat{\exp (i b / 2)}$ is the quantum operator corresponding to the exponential version of $b$. Moreover, $\{|v\rangle\}$ is the basis of eigenstates of the volume operator $\hat{v}$ satisfying $\left\langle v \mid v^{\prime}\right\rangle=\delta_{v, v^{\prime}}$. On this quantum background, the gravitational Hamiltonian constraint becomes [10, 27]

$$
\hat{H}_{\mathrm{gr}}=-\frac{3 \pi G}{8 \alpha} \sqrt{|\hat{v}|}\left(\hat{\mathcal{N}}-\hat{\mathcal{N}}^{-1}\right)^{2} \sqrt{|\hat{v}|} .
$$

In the absence of external fields, physical states must satisfy

$$
i \hbar \partial_{T} \Psi_{o}(v, T)=\hat{\mathcal{H}}_{\mathrm{gr}} \Psi_{o}(v, T),
$$

where we have replaced $\Psi$ by the pure gravitational states $\Psi_{o} \in \mathscr{H}_{\text {gr }}$ only. These states provide the scalar product with a finite norm

$$
\left\langle\Psi_{o} \mid \Psi_{o}^{\prime}\right\rangle=\sum_{v} \Psi_{o}^{*}\left(v, T_{0}\right) \Psi_{o}^{\prime}\left(v, T_{0}\right),
$$

and belong to the physical Hilbert space $\mathscr{H}_{\text {Phys }}^{o}$ of the geometry. Here, $T_{0}$ is any "instant" of relational time $T$. We will set the norm of the scalar product of the gravitational states to be $\left\langle\Psi_{o} \mid \Psi_{o}\right\rangle=1$.

As we can see from Eq. (3.10), the background geometry is quantized due to quantum gravity, so the classical geometrical variables in the matter sector (2.24) should be replaced by the corresponding quantum operators on $\mathscr{H}_{\text {gr }}$. Therefore, $\hat{H}_{\mathrm{EM}}$ in Eq. (3.10) can be written as

$$
\hat{H}_{\mathrm{EM}} / \ell^{3}=: \sum_{\mathbf{k} \in \mathcal{L}} \sum_{r}^{2} \hat{H}_{T, \mathbf{k}}^{(r)},
$$

where

$$
\hat{H}_{T, \mathbf{k}}^{(r)}:=\frac{1}{2 \ell^{3}}\left[\widehat{a^{-3}} \otimes\left(\hat{P}_{\mathbf{k}}^{r}\right)^{2}+k^{2} \hat{a} \otimes\left(\hat{Q}_{\mathbf{k}}^{r}\right)^{2}\right] .
$$

Then, from Eqs. (3.15) and (3.10), the Schrödinger equation for each mode and polarization of the EM field is written as

$$
\begin{aligned}
i \hbar \partial_{T} \Psi(v, Q, T) & =\left[\hat{\mathcal{H}}_{\mathrm{gr}}+\hat{H}_{T, \mathbf{k}}^{(r)}\right] \Psi(v, Q, T) \\
& =: \hat{\tilde{\mathcal{H}}}_{\mathbf{k}} \Psi(v, Q, T)
\end{aligned}
$$

where $\hat{\tilde{\mathcal{H}}}_{\mathbf{k}}$ is the physical Hamiltonian for each mode.

\section{EMERGING GEOMETRIES}

For the test field propagating on the quantized background provided in the previous section, we apply two approximations in order to predict the emerging effective background geometry: (i) the test field approximation in which backreaction is discarded (see Sec. IVA below), and (ii) the Born-Oppenheimer approximation in which the backreaction is present (see Sec. IVB below). Then, we discuss the phenomenological features of those two geometries (see Sec. IVC).

\section{A. Test-field approximation}

The evolution equation (3.17) is rather analogous to the Schrödinger equation (2.27). However, the quantum geometry operators in $\hat{\mathcal{H}}_{\mathrm{gr}}$ do not depend on time, and Eq. (3.17) provides quantum evolution for the state $\Psi(v, Q, T)$ that depends on the $\mathbf{k t h}$ mode of the test EM field and the quantum geometry, while Eq. (2.27) evolves the state $\psi(Q, T)$ (of the EM test field only) on the (timedependent) classical FLRW background. To make two evolutions comparable, we should work in the "interaction picture" [4] by setting

$$
\Psi(v, Q, T)=e^{-(i / \hbar) \hat{H}_{o}\left(T-T_{0}\right)} \Psi_{\mathrm{int}}(v, Q, T)
$$

for any instant of relational time $T_{0}$. Then, Eq. (3.17) reduces to

$$
\begin{aligned}
i \hbar \partial_{T} \Psi_{\mathrm{int}}=\frac{1}{2 \ell^{3}}\left[\widehat{a^{-3}}(T) \otimes\left(\hat{P}_{\mathbf{k}}^{r}\right)^{2}\right. & \\
& \left.+k^{2} \hat{a}(T) \otimes\left(\hat{Q}_{\mathbf{k}}^{r}\right)^{2}\right] \Psi_{\text {int }},
\end{aligned}
$$


where any operator $\hat{A}(T)$ is the corresponding timedependent operator in the Heisenberg picture now:

$$
\hat{A}(T)=e^{\frac{i}{\hbar} \hat{H}_{o}\left(T-T_{0}\right)} \hat{A} e^{-\frac{i}{\hbar} \hat{H}_{o}\left(T-T_{0}\right)} .
$$

In this picture, the quantum geometry (presented by the heavy degrees of freedom $\hat{\mathcal{H}}_{\mathrm{gr}}$ in the total Hamiltonian) is in effect described in the Heisenberg picture (i.e., its states are frozen at time $T_{0}$, but the scale factor operators evolve in time), while the test field (as a perturbation denoted as light degrees of freedom, provided by $\hat{H}_{T, \mathbf{k}}^{(r)}$ ) is described using the Schrödinger picture.

When a test-field approximation is considered, i.e., when a matter backreaction is discarded, then, for any time $T$, one can decompose the total wave function as [4]

$$
\Psi_{\text {int }}(v, Q, T)=\Psi_{o}\left(v, T_{0}\right) \otimes \psi(Q, T)
$$

so that the geometry's quantum state $\Psi_{o}(v, T)$ obeys the "unperturbed" evolution equation $i \hbar \partial_{T} \Psi_{o}(v, T)=$ $\hat{\mathcal{H}}_{\mathrm{gr}} \Psi_{o}(v, T)$. In this case, the Schrödinger equation (3.17) reduces to an evolution equation for $\psi(Q, T)$ only:

$$
i \hbar \partial_{T} \psi=\frac{1}{2 \ell^{3}}\left[\left\langle\widehat{a^{-3}}(T)\right\rangle_{o}\left(\hat{P}_{\mathbf{k}}^{r}\right)^{2}+k^{2}\langle\hat{a}(T)\rangle_{o}\left(\hat{Q}_{\mathbf{k}}^{r}\right)^{2}\right] \psi .
$$

By comparison with Eq. (2.27), Eq. (4.5) can be seen as $\mathrm{a}(\mathrm{n})$ (Schrödinger) evolution equation for the kth mode of the EM field on a dressed spacetime with $\left(M, \bar{g}_{a b}\right)$,

$$
\bar{g}_{a b} d x^{a} d x^{b}=-\bar{N}_{T}^{2}(T) d T^{2}+\bar{a}^{2}(T) d \mathbf{x}^{2},
$$

where the metric components $\bar{N}_{T}$ and $\bar{a}$ have relations with the expectation values of the original spacetime operators as

$$
\bar{N}_{T} / \bar{a}^{3}=\ell^{-3}\left\langle\widehat{a^{-3}}(T)\right\rangle_{o}, \quad \bar{N}_{T} \bar{a}=\ell^{-3}\langle\hat{a}(T)\rangle_{o} .
$$

Equation (4.7) provides two equations for two unknown variables of the emergent dressed background metric (4.6). Using these two equations, we find that

$$
\begin{aligned}
\bar{N}_{T}(T) & =\ell^{-3}\left[\left\langle\widehat{a^{-3}}(T)\right\rangle_{o}\langle\hat{a}(T)\rangle_{o}^{3}\right]^{\frac{1}{4}}, \\
\bar{a}(T) & =\left[\frac{\langle\hat{a}(T)\rangle_{o}}{\left\langle\widehat{a^{-3}}(T)\right\rangle_{o}}\right]^{\frac{1}{4}} .
\end{aligned}
$$

The emerging geometry (4.6), provided by the components (4.8), does not depend on any specific chosen mode of the test EM field, and therefore, there is no violation of the Lorentz symmetry. However, for a massive field (such as the Proca field), one may expect a mode-dependent solution such that a rainbow metric can emerge for the effective background geometry [13].

\section{B. Born-Oppenheimer approximation}

So far, we have seen that, when the backreaction of the EM field on the background quantum geometry is discarded due to a test-field approximation procedure, no deviation from the local Lorentz symmetry emerges, and the dressed background metric is independent of the chosen EM field mode. However, it is possible to lift this condition by employing a Born-Oppenheimer approximation scheme in the evolution equation (4.2).

The Born-Oppenheimer approximation is a particular form of a general method called the adiabatic approximation, which underlines the theory of molecular motion in atomic physics, based on a general assumption that the heavy degrees of freedom (such as nuclei) evolve so slowly compared with the light degrees of freedom (i.e., the electron in the atom) that the force between the atoms can still be calculated by differentiation of the energies $E_{e}^{(l)}$ of each perturbation's (i.e., electronic) eigenstates $l$. The function $E_{e}^{(l)}$ thus serves as the potential energy for the evolution of the nuclei if the electrons are in state $l$. An extension of this approximation has also been made in the context of quantum gravity, especially in the WheelerdeWitt (see Ref. 28] and references therein) and LQG approaches [29, 30].

As we have seen before, the dynamics of the gravityfield system is generated by the physical Hamiltonian (3.17). Here, the gravitational sector of the Hamiltonian (3.17) represents the heavy degrees of freedom, and the field Hamiltonian represents the light degrees of freedom. One difference to the Hamiltonian in a hydrogen atom is that, here, the light degrees of freedom (i.e., the EM field) are not only the functions of field variable $(Q, P)$ but are functions of the gravitational variable $\hat{v}$ as well. Our task will be then to obtain solutions to the full eigenvalue problem,

$$
\begin{aligned}
\left\{\hat{\mathcal{H}}_{\mathrm{gr}}\right. & +\frac{1}{2 \ell^{3}}\left[\widehat{a^{-3}} \otimes\left(\hat{P}_{\mathbf{k}}^{r}\right)^{2}\right. \\
& \left.\left.+k^{2} \hat{a} \otimes\left(\hat{Q}_{\mathbf{k}}^{r}\right)^{2}\right]\right\} \tilde{\Psi}(v, Q)=E_{\mathbf{k}} \tilde{\Psi}(v, Q),
\end{aligned}
$$

where $E_{\mathbf{k}}$ is the total energy eigenvalue of each mode.

The Born-Oppenheimer approximation consists of assuming that the solution to (4.9) has the form

$$
\tilde{\Psi}(v, Q)=\sum_{\mu, l} c_{\mu l} \xi_{l}^{\mu}(v) \otimes \chi^{l}(v, Q),
$$

for the stationary states $\tilde{\Psi} \in \mathscr{H}\left(=\mathscr{H}_{\mathrm{gr}} \otimes \mathscr{H}_{r, \mathbf{k}}\right)$, where $c_{\mu l}$ are expansion coefficients. For the wave function (4.10), the Schrödinger equation (3.17) is separated into a stationary state equation for the field mode,

$$
\hat{H}_{T, \mathbf{k}}^{(r)} \chi^{l}(Q ; v)=\epsilon_{\mathbf{k}}^{(l)}(\hat{v}) \chi^{l}(Q ; v),
$$

on the specified (fixed) background quantum geometry ${ }^{4}$

\footnotetext{
4 That is, the dependence on gravitational variable $v$ has to be understood in a parametrical way so that we assume that $v$ is fixed and regard $\chi^{l}(Q ; v)$ as an element on $\mathscr{H}_{r, \mathbf{k}}$. In a sense, this means we can solve Eq. 4.11 for each external parameter $Q$ separately.
} 
with the state $\xi_{l}^{\mu}(v)$, and a second equation describing the evolution of the background:

$$
\left[\hat{\mathcal{H}}_{\mathrm{gr}}+\lambda \epsilon_{\mathbf{k}}^{(l)}(\hat{v})\right] \xi_{l}^{\mu}(v)=E_{l}^{\mu} \xi_{l}^{\mu}(v) .
$$

Here, $\lambda$ is a real constant introduced to keep track of the number of times the perturbation enters, and $\epsilon_{\mathbf{k}}^{(l)}(\hat{v})$ is the eigenvalue of the $l$ th eigenstate of the field mode (light variable), but it is still an operator on the quantum geometrical Hilbert space. Now, due to the orthonormality of $\chi^{l}$, we get

$$
\begin{aligned}
\epsilon_{\mathbf{k}}^{(l)}(\hat{v})=\frac{1}{2 \ell^{3}}\left[\widehat{a^{-3}}\right. & \left\langle\chi^{l}\left|\left(\hat{P}_{\mathbf{k}}^{r}\right)^{2}\right| \chi^{l}\right\rangle \\
& \left.+k^{2} \hat{a}\left\langle\chi^{l}\left|\left(\hat{Q}_{\mathbf{k}}^{r}\right)^{2}\right| \chi^{l}\right\rangle\right] .
\end{aligned}
$$

Note that $\xi_{l}^{\mu}$ is the eigenstate of the (backreacted) geometry due to the presence of the $l$ th eigenstate of the EM field, which is different from unperturbed eigenstates $\xi_{o}^{\mu}$. If $\lambda \epsilon_{\mathrm{k}}^{(l)}$ in Eq. (4.12) were negligible, then the BornOppenheimer description here reduces to the test field approximation, in which the gravitational sector does not know anything about the field modes and the gravitational wave function is simply given by the solution to $\hat{\mathcal{H}}_{\mathrm{gr}} \xi_{o}^{\mu}(v)=E_{o}^{\mu} \xi_{o}^{\mu}(v)$ for which $E_{l}^{\mu}=E_{o}^{\mu}$. However, in the case $\lambda \epsilon_{\mathbf{k}}^{(l)} \neq 0$ here, the field mode influences the heavy degrees of freedom effectively, and $\xi_{l}^{\mu} \neq \xi_{o}^{\mu}$ (although it is not taken into account with respect to full dynamics).

After analyzing the eigenvalue equation (4.11) for the field mode, we can find the eigenfunctions $\chi^{l}(Q ; v)$ and eigenvalues $\epsilon_{\mathrm{k}}^{(l)}(\hat{v})$. Then, by substituting these functions in Eq. (4.12), we can compute the coefficients $\xi_{l}^{\mu}(v)$, and thus we will find the complete solutions (4.10) to the full quantum theory of EM modes on the quantum FLRW background. To do so, we employ perturbation theory in order to compute approximate solution for the eigenstates $\xi_{l}^{\mu}$. In this mechanism, since $\left\{\xi_{o}^{\mu}\right\}$ form a complete set of bases for the unperturbed wave function $\Psi_{o}$ (with the eigenvalues $E_{o}^{\mu}$ ), we can expand the perturbed states $\xi_{l}^{\mu}$ in terms of them as well,

$$
\xi_{l}^{\mu}=N(n)\left[\xi_{o}^{\mu}+\sum_{\nu \neq \mu} \sum_{n} \lambda^{n} \beta_{\mu \nu}^{n(l)} \xi_{o}^{\nu}\right]
$$

in which $\beta_{\mu \nu}^{n(l)}$ (with $n \neq 0$ ) are the expansion coefficients for the $n$th order of perturbation. By substituting (4.14) in the Schrödinger equation (4.12), we can find the coefficients $\beta_{\mu \nu}^{n(l)}$ and the energy eigenvalues $E_{l}^{\mu}=E_{o}^{\mu}+\lambda E_{l}^{\mu(1)}+\lambda^{2} E_{l}^{\mu(2)}+\cdots$. To the first order of perturbation (i.e., for $n=1$ ), we get

$$
\beta_{\mu \nu}^{1(l)}:=\frac{\epsilon_{\mu \nu}^{(l)}(k)}{E_{o}^{\mu}-E_{o}^{\nu}}
$$

where

$$
\begin{aligned}
\epsilon_{\mu \nu}^{(l)}:= & \left\langle\xi_{o}^{\nu}\left|\epsilon_{\mathbf{k}}^{(l)}(\hat{v})\right| \xi_{o}^{\mu}\right\rangle \\
= & \frac{1}{2 \ell^{3}}\left[\left\langle\xi_{o}^{\nu}\left|\widehat{a^{-3}}\right| \xi_{o}^{\mu}\right\rangle\left\langle\chi^{l}\left|\left(\hat{P}_{\mathbf{k}}^{r}\right)^{2}\right| \chi^{l}\right\rangle\right. \\
& \left.\quad+k^{2}\left\langle\xi_{o}^{\nu}|\hat{a}| \xi_{o}^{\mu}\right\rangle\left\langle\chi^{l}\left|\left(\hat{Q}_{\mathbf{k}}^{r}\right)^{2}\right| \chi^{l}\right\rangle\right] \\
= & : A_{\mu \nu}^{(l)}+k^{2} B_{\mu \nu}^{(l)} .
\end{aligned}
$$

Moreover, the normalization coefficient $N(n)$ is chosen such that $N(0)=1$ and $\lambda=0$ for the unperturbed case. The first-order corrections to the energy are then given by

$$
E_{l}^{\mu}=E_{o}^{\mu}+\lambda\left\langle\xi_{o}^{\mu}\left|\epsilon_{\mathbf{k}}^{(l)}(\hat{v})\right| \xi_{o}^{\mu}\right\rangle+\mathcal{O}\left(\lambda^{2}\right) .
$$

By setting $\left\langle\xi_{l}^{\mu} \mid \xi_{l}^{\mu}\right\rangle=1$, we obtain $N(1)=1$ up to the first-order corrections. Then, $\xi_{l}^{\mu}$ becomes

$$
\xi_{l}^{\mu}=\xi_{o}^{\mu}+\lambda \sum_{\nu \neq \mu} \beta_{\mu \nu}^{1(l)} \xi_{o}^{\nu} .
$$

To have better insight regarding the evolution of the field modes on the herein quantum background, when the coefficients $\xi_{l}^{\mu}$ are already known through Eq. (4.18), similar to the case we had in the test-field approximation, we will describe the situation in a way in which the background geometry can be treated almost classically. By substituting Eq. (4.18) in the total wave function (4.10), we obtain

$$
\begin{aligned}
\tilde{\Psi}(v, Q) & =\sum_{\mu, l}\left[c_{\mu}^{o} \xi_{o}^{\mu} \otimes b_{l} \chi^{l}+\lambda \sum_{\nu \neq \mu} c_{\mu l} \beta_{\mu \nu}^{1(l)} \xi_{o}^{\nu} \otimes \chi^{l}\right] \\
& =: \Psi_{o}(v) \otimes \psi(Q)+\delta \Psi(v, Q),
\end{aligned}
$$

in which the first term (on the right-hand side) denotes the unperturbed wave function and the second term denotes the influence of the light degrees of freedom (i.e., EM mode perturbation) on the geometry quantum state. In particular, we are interested in a description of quantum modes on a(n) (effective) classical background, so we will further assume that the field eigenstates $\chi^{l}$ are not affected by quantum geometry, i.e., they are not entangled with the background eigenstates $\xi_{l}^{\mu}$. Then, we can decompose the perturbation wave function $\mathrm{as}^{5}$

$$
\delta \Psi(v, Q) \approx \delta \Psi_{o}(v) \otimes \psi(Q),
$$

5 The backreaction term in Eq. 4.19) can be decomposed as

$$
\begin{aligned}
\delta \Psi(v, Q) & =\lambda \sum_{\mu, l} c_{\mu l} \sum_{\nu \neq \mu} \beta_{\mu \nu}^{1(l)} \xi_{o}^{\nu} \otimes \chi^{l} \\
& =: \lambda \sum_{\mu} \sum_{\nu \neq \mu} \tilde{c}_{\mu}^{o} \beta_{\mu \nu}^{1} \xi_{o}^{\nu} \otimes \sum_{l} \tilde{b}_{l} \chi^{l} .
\end{aligned}
$$

Notice that the last relation above was obtained by assuming that $c_{\mu l}=\tilde{c}_{\mu}^{o} \tilde{b}_{l}$, and $\beta_{\mu \nu}^{1(l)}$ is the same for all $\chi^{l}$, such that $\beta_{\mu \nu}^{1(l)} \equiv \beta_{\mu \nu}^{1}$. In other words, decomposition 4.20 is only relevant when the field eigenstates are degenerate. 
where

$$
\delta \Psi_{o}(v)=\lambda \sum_{\mu} \sum_{\nu \neq \mu} c_{\mu}^{o} \beta_{\mu \nu}^{1} \xi_{o}^{\nu} .
$$

Now, by substituting the total (perturbed) wave function (4.19), with the disentangled perturbation term $\delta \Psi=$ $\delta \Psi_{o} \otimes \psi$ above, into the total time-dependent Schrödinger equation (3.17), we obtain

$$
\begin{aligned}
i \hbar \partial_{T} \psi(Q, T)=\frac{1}{2 \ell^{3}} & {\left[\left(\widehat{\left\langle a^{-3}\right.}\right\rangle_{o}+\lambda\left\langle\widehat{a^{-3}}\right\rangle_{\delta}\right)\left(\hat{P}_{\mathbf{k}}^{r}\right)^{2} } \\
& \left.+k^{2}\left(\langle\hat{a}\rangle_{o}+\lambda\langle\hat{a}\rangle_{\delta}\right)\left(\hat{Q}_{\mathbf{k}}^{r}\right)^{2}\right] \psi
\end{aligned}
$$

Here, we have defined $\left\langle\widehat{a^{-3}}\right\rangle_{\delta} \equiv\left\langle\Psi_{o}\left|\widehat{a^{-3}}\right| \delta \Psi_{o}\right\rangle$ and $\langle\hat{a}\rangle_{\delta} \equiv$ $\left\langle\Psi_{o}|\hat{a}| \delta \Psi_{o}\right\rangle$. We have further used the fact that the perturbed wave function $\delta \Psi_{o}$ is orthogonal to $\Psi_{o}$, so $\left\langle\Psi_{o} \mid \delta \Psi_{o}\right\rangle=0$. To make a comparison between the backreacted quantum geometry implemented by Eq. (4.22) and the dressed background (4.5) provided by the testfield approximation, it is convenient to expand correction terms in Eq. (4.22), in terms of the unperturbed background eigenstates $\xi_{o}^{\mu}$, as

$$
\begin{aligned}
\left\langle\widehat{a^{-3}}\right\rangle_{\delta} & =\sum_{\sigma, \mu} \sum_{\nu \neq \mu}\left(c_{\sigma}^{o}\right)^{*} c_{\mu}^{o} \frac{\left\langle\xi_{o}^{\sigma}\left|\widehat{a^{-3}}\right| \xi_{o}^{\nu}\right\rangle}{E_{o}^{\mu}-E_{o}^{\nu}} \epsilon_{\mu \nu}^{(l)}(k), \\
\langle\hat{a}\rangle_{\delta} & =\sum_{\sigma, \mu} \sum_{\nu \neq \mu}\left(c_{\sigma}^{o}\right)^{*} c_{\mu}^{o} \frac{\left\langle\xi_{o}^{\sigma}|\hat{a}| \xi_{o}^{\nu}\right\rangle}{E_{o}^{\mu}-E_{o}^{\nu}} \epsilon_{\mu \nu}^{(l)}(k) .
\end{aligned}
$$

Since $\left\langle\widehat{a^{-3}}\right\rangle_{\delta}$ and $\langle\hat{a}\rangle_{\delta}$ are functions of $\epsilon_{\mu \nu}(k)$, these coefficients depend on the mode $\mathbf{k}$ of the EM field. Now, as before, by comparison with Eq. (2.27), Eq. (4.22) can be seen as an evolution equation for $\mathbf{k t h}$ mode of the EM field on a dressed spacetime with $\left(M, \tilde{g}_{a b}\right)$,

$$
\tilde{g}_{a b} d x^{a} d x^{b}=-\tilde{N}_{T}^{2}(T) d T^{2}+\tilde{a}^{2}(T) d \mathbf{x}^{2}
$$

where $\tilde{N}_{T}$ and $\tilde{a}$ are related to the expectation values of the original spacetime operators as

$$
\begin{aligned}
\tilde{N}_{T} / \tilde{a}^{3} & =\ell^{-3}\left[\left\langle\widehat{a^{-3}}\right\rangle_{o}+\lambda\left\langle\widehat{a^{-3}}\right\rangle_{\delta}\right] \\
\tilde{N}_{T} \tilde{a} & =\ell^{-3}\left[\langle\hat{a}\rangle_{o}+\lambda\langle\hat{a}\rangle_{\delta}\right] .
\end{aligned}
$$

Solving the equations above, we obtain

$$
\begin{aligned}
\tilde{N}_{T}= & \ell^{-3}\left[\left(\left\langle\widehat{a^{-3}}\right\rangle_{o}+\lambda\left\langle\widehat{a^{-3}}\right\rangle_{\delta}\right)\right. \\
& \left.\times\left(\langle\hat{a}\rangle_{o}+\lambda\langle\hat{a}\rangle_{\delta}\right)^{3}\right]^{\frac{1}{4}}, \\
\tilde{a}(T)= & {\left[\frac{\langle\hat{a}\rangle_{o}+\lambda\langle\hat{a}\rangle_{\delta}}{\left\langle\widehat{a^{-3}}\right\rangle_{o}+\lambda\left\langle\widehat{a^{-3}}\right\rangle_{\delta}}\right]^{\frac{1}{4}} . }
\end{aligned}
$$

By expanding the relations above, we can rewrite them as

$$
\begin{aligned}
\tilde{N}_{T} & =\bar{N}_{T}[1+\lambda \beta(k)], \\
\tilde{a}(T) & =\bar{a}(T)[1+\lambda \sigma(k)],
\end{aligned}
$$

where $\bar{N}_{T}$ and $\bar{a}$ are given by Eq. (4.8) and $\beta(k)$ and $\sigma(k)$ are defined as

$$
\begin{aligned}
& \beta(k)=\frac{1}{4} \frac{\left.\widehat{a^{-3}}\right\rangle_{\delta}}{\left\langle\widehat{a^{-3}}\right\rangle_{o}}+\frac{3}{4} \frac{\langle\hat{a}\rangle_{\delta}}{\langle\hat{a}\rangle_{o}}+\cdots \\
& \sigma(k)=\frac{1}{4} \frac{\langle\hat{a}\rangle_{\delta}}{\langle\hat{a}\rangle_{o}}-\frac{1}{4} \frac{\left.\widehat{a^{-3}}\right\rangle_{\delta}}{\left\langle\widehat{a^{-3}}\right\rangle_{o}}+\cdots
\end{aligned}
$$

This indicates that in the presence of the backreaction the components of the effective metric (4.25) depend on the mode of the field. Thus, each mode of the EM field probes a dressed background spacetime differently from the other modes, so the effective background looks like a rainbow metric (see, for example, Refs. [21, 22]) from the field's point of view.

\section{Rainbow metric: Violation of the local Lorentz symmetry}

So far, we have seen that when the energy $\epsilon_{\mathbf{k}}^{(l)}$ of the mode's eigenstate $\chi^{l}$ is high the effective background probed by the mode becomes $k$ dependent, due to the backreaction effects predicted by the Born-Openheimer approximation. Now, we provide an interpretation for a "classical observer" measuring the background metric $\bar{g}_{a b}$ probed by low-energy modes [see Eq. [4.6)], while the high-energy modes propagate on $\tilde{g}_{a b}(k)$ [given by Eq. (4.25)].

In general, a (classical) cosmological observer with a normalized 4-velocity $u^{a}=\left(1 / \bar{N}_{T}, 0,0,0\right)$ measures the energy of a particle with the 4-momentum $k_{a}=$ $\left(k_{0}, k_{1}, k_{2}, k_{3}\right)$ to be $E=k_{a} u^{a}$. The normalization condition for the 4 -velocity implies $\bar{g}_{a b} u^{a} u^{b}=-1$. For the emerging background (4.25), the on-shell relation for the photon with the mass $m_{0}=0$ becomes $\tilde{g}^{a b} k_{a} k_{b}=0$ so that

$$
\begin{aligned}
\tilde{\omega}_{k}^{2}(k) & =k_{0}^{2}=\frac{\bar{N}_{T}^{2}}{\bar{a}^{2}} k^{2}\left(\frac{1+\lambda \beta(k)}{1+\lambda \sigma(k)}\right)^{2} \\
& =: f^{2}(k) \bar{\omega}_{k}^{2}(k) .
\end{aligned}
$$

This indicates that from the point of view of the classical cosmological observer on the backreacted background (4.25) the energy $\bar{\omega}_{k}$ of the EM modes is modified by a mode-dependent function, $f(k) \neq 1$.

The appropriate rescaled components of the physical momentum $p$ and the energy $E$ in the tetrad frame of the classical observer (where $\bar{g}_{a b}=\eta_{A B} e_{a}^{A} e_{b}^{B}$, with the internal indices $A, B=0,1,2,3$ and the internal metric $\eta_{A B}$ such that $\eta^{A B}=e^{A a} e_{a}^{B}$ ) are given by

$$
k_{\hat{0}}=\frac{\tilde{\omega}}{\bar{N}_{T}}=: E, \quad \text { and } \quad k_{I}=\frac{k_{i}}{\bar{a}},
$$

where $k_{A}=e_{A}^{a} k_{a}, p^{2}=k_{I} k^{I}$ (with $\hat{0}$ denoting the zeroth component of the internal metric and $I, J=1,2,3$ 
being the three-dimensional internal indices). Then, the 3 -velocity $v^{I}$ of the photon measured in the threedimensional internal basis of the cosmological observer reads

$$
v^{I}=\frac{d E}{d k_{I}},
$$

with the squared norm $|v|^{2}=v_{I} v^{I}$, such that

$$
|v| \approx 1+\lambda\left(L_{1}^{(l)}+3 L_{2}^{(l)} p^{2}\right)+\mathcal{O}\left(p^{4}, \lambda^{2}\right) .
$$

Here, $L_{1}^{(l)}$ and $L_{2}^{(l)}$ are functions of quantum fluctuation of the background geometry and the field mode,

$$
\begin{aligned}
L_{1}^{(l)} & =\frac{1}{2} \sum_{\sigma, \mu} \sum_{\nu \neq \mu} \frac{\left(c_{\sigma}^{o}\right)^{*} c_{\mu}^{o} Z_{\sigma \nu}}{E_{o}^{\mu}-E_{o}^{\nu}} A_{\mu \nu}^{(l)}, \\
L_{2}^{(l)} & =\frac{\bar{a}^{2}}{2} \sum_{\sigma, \mu} \sum_{\nu \neq \mu} \frac{\left(c_{\sigma}^{o}\right)^{*} c_{\mu}^{o} Z_{\sigma \nu}}{E_{o}^{\mu}-E_{o}^{\nu}} B_{\mu \nu}^{(l)},
\end{aligned}
$$

where

$$
Z_{\sigma \nu}:=\left[\frac{\left\langle\xi_{o}^{\sigma}\left|\widehat{a^{-3}}\right| \xi_{o}^{\nu}\right\rangle}{\left\langle\widehat{a^{-3}}\right\rangle_{o}}+\frac{\left\langle\xi_{o}^{\sigma}|\hat{a}| \xi_{o}^{\nu}\right\rangle}{\langle\hat{a}\rangle_{o}}\right],
$$

and $A_{\mu \nu}^{(l)}$ and $B_{\mu \nu}^{(l)}$ are defined in Eq. (4.16). In the presence of $L_{1}^{(l)}, L_{2}^{(l)} \neq 0$ in Eq. (4.37), it is seen that EM modes propagate with the speed higher than the speed of light $(c=1)$ on the background (4.25). By expanding Eq. (4.34), we get

$$
E^{2} \approx\left(1+2 \lambda L_{1}^{(l)}\right) p^{2}+2 \lambda L_{2}^{(l)} p^{4}+\mathcal{O}\left(p^{5}, \lambda^{2}\right) .
$$

Thus, the dispersion relation on the effective background (4.25) deviates from the standard dispersion relation $E^{2}=p^{2}$. This deviation is held even in the low-energy limit (with $k \ll 1$ ) since $L_{1}^{(l)} \neq 0$ or when the EM field mode state (denoted by $l$ ) is only a vacuum state in the Fock space. These analyzes indicate that the local Lorentz symmetry is violated on the effective geometry (4.25). The standard dispersion relation is recovered only when the quantum gravity induced parameters $L_{1}^{(l)}, L_{2}^{(l)}$ vanish.

\section{PARTICLE PRODUCTION ON EMERGING BACKGROUND}

In this section, we are interested in studying how those quantum gravity effects, in the presence of the field's backreaction [leading to an emergent rainbow geometry [4.25] ], can give rise to the creation of the quantum particles from the Planck regime ${ }^{6}$.

\footnotetext{
${ }^{6}$ For the issue of particle production in quantum cosmology, see for example Ref. 31]
}

The Hamiltonian of the EM field for a background metric (4.25) can be written as

$$
i \hbar \partial_{T} \psi=\frac{\tilde{N}_{T}}{2 \tilde{a}^{3}}\left[\left(\hat{P}_{\mathbf{k}}^{r}\right)^{2}+k^{2} \tilde{a}^{4}\left(\hat{Q}_{\mathbf{k}}^{r}\right)^{2}\right] \psi .
$$

When the background metric has no dependence on the wave number $k$ (i.e., for $\beta=\sigma=0$ ), then $\tilde{N}_{T}=\bar{N}_{T}$, $\tilde{a}=\bar{a}$, and Eq. (5.1) reduces to the Hamiltonian equation (4.5). For nonvanishing backreaction effects, $\beta(k) \neq 0$ and $\sigma(k) \neq 0$, the corresponding (classical) equation of motion (2.28) for Eq. (5.1) is modified as

$$
\ddot{Q}_{\mathbf{k}}^{r}+\frac{\dot{\tilde{m}}}{\tilde{m}} \dot{Q}_{\mathbf{k}}^{r}+\tilde{\omega}_{k}^{2}(T) Q_{\mathbf{k}}^{r}=0
$$

in which a dot, here, denotes a derivative with respect to the internal time $T$. Now, the frequency of each mode $\tilde{\omega}_{k}(T)$ is corrected by the $k$-dependent term $f(k)$, as given in Eq. (4.34), and $\tilde{m}$ is given by

$$
\tilde{m}=\frac{\tilde{a}^{3}}{\tilde{N}_{T}}=\frac{\bar{a}^{3}}{\bar{N}_{T}} \frac{(1+\lambda \sigma(k))^{3}}{(1+\lambda \beta(k))} .
$$

This equation indicates that $\dot{\tilde{m}} / \tilde{m}$ in Eq. (5.2) cannot vanish anymore, because $\tilde{N}_{T} / \tilde{a}^{3} \neq 1$. To study the particle creation mechanism using the approach presented in Ref. [32], it is convenient that the equation of motion (5.2) takes the form of Eq. (2.29). To do so, we cannot use the usual harmonic time gauge technique as we had at the end of Sec. [I] since $\dot{\tilde{m}} \neq 0$ on the emerging effective metric 4.25). However, we can consider any new time coordinate $\tilde{\tau}$ (with a lapse function $N_{\tilde{\tau}}$ ) such that $N_{\tilde{\tau}} d \tilde{\tau}=\tilde{N}_{T} d T$. Then, evolution in terms of the new time parameter $\tilde{\tau}$ is given by the $\tilde{\tau}$-dependent Schrödinger equation:

$$
i \hbar \partial_{\tilde{\tau}} \psi=\frac{\tilde{N}_{\tilde{\tau}}}{2 \tilde{a}^{3}}\left[\left(\hat{P}_{\mathbf{k}}^{r}\right)^{2}+k^{2} \tilde{a}^{4}\left(\hat{Q}_{\mathbf{k}}^{r}\right)^{2}\right] \psi .
$$

Without loss of generality, we shall consider the lapse function $N_{\tilde{\tau}}$ such that $\tilde{\tau}$ is a harmonic time, i.e., $N_{\tilde{\tau}}=\tilde{a}^{3}$. In this time gauge, we have that

$$
d \tilde{\tau}=\frac{\tilde{N}_{T}}{\tilde{a}^{3}} d T=\frac{\bar{N}_{T}}{\bar{a}^{3}} \frac{(1+\lambda \beta(k))}{(1+\lambda \sigma(k))^{3}} d T .
$$

In terms of this new (harmonic) time coordinate $\tilde{\tau}$, the classical equation of motion corresponding to the Schrödinger equation (5.4) becomes

$$
Q_{\mathbf{k}}^{r^{\prime \prime}}+\tilde{\omega}_{k}^{2}(\tilde{\tau}) Q_{\mathbf{k}}^{r}=0,
$$

in which a prime now denotes a derivative with respect to the harmonic time $\tilde{\tau}$ and $\tilde{\omega}_{k}$ is given by

$$
\tilde{\omega}_{k}^{2}(T)=k^{2} \bar{a}^{4}[1+\lambda \sigma(k)]^{4}=k^{2} \tilde{a}^{4} .
$$

There exists a complete set of solutions to Eq. (5.4) characterized by the quantum number $n$ as [32, 33]

$$
\chi_{n}(Q, \tilde{\tau})=\frac{\left(v_{k}^{r *}\right)^{\frac{n}{2}} \mathrm{H}_{n}\left(\frac{Q_{k}^{r}}{\sqrt{2}\left|v_{k}^{r}\right|}\right) \exp \left[i v_{k}^{r^{\prime}} \frac{\left(Q_{k}^{r}\right)^{2}}{2 v_{k}^{r}}\right]}{2^{\frac{n}{2}}(2 \pi)^{\frac{1}{4}}(n !)^{\frac{1}{2}}\left(v_{k}^{r}\right)^{\frac{n+1}{2}}},
$$


where $v_{k}^{r}(\tilde{\tau})$ is a solution of Eq. (5.6) and $\mathrm{H}_{n}$ is the Hermite polynomial of order $n$. The states (5.8) can be generated by defining creation and annihilation operators $\hat{A}_{\mathrm{k}}^{\dagger}$ and $\hat{A}_{\mathbf{k}}$ 34],

$$
\hat{A}_{\mathbf{k}}=-i v_{k}^{r^{\prime}}(\tilde{\tau}) \hat{Q}_{\mathbf{k}}^{r}+v_{k}^{r}(\tilde{\tau})\left(\partial / \partial Q_{\mathbf{k}}^{r}\right),
$$

where $\hat{A}_{\mathrm{k}}^{\dagger}$ is its Hermitian conjugate of (5.9). These operators have the properties

$$
\chi_{n}=(n !)^{-1 / 2}\left(\hat{A}_{\mathbf{k}}^{\dagger}\right)^{n} \chi_{0}, \quad \hat{A}_{\mathbf{k}} \chi_{0}=0,
$$

where $\left[\hat{A}_{\mathbf{k}}, \hat{A}_{\mathbf{k}}^{\dagger}\right]=1$.

Let us assume (following Zel'dovich [35]) that there is a regime $\tilde{\tau} \leq \tilde{\tau}_{i}$ such that the vacuum state satisfies an adiabatic condition. Then, we can construct the usual harmonic oscillator states and choose $|0\rangle$ as the vacuum wave function defined by

$$
\frac{1}{2} \tilde{\omega}_{k}\left(\tilde{\tau}_{i}\right)|0\rangle=\hat{H}_{\tilde{\tau}, \mathbf{k}}^{(r)}\left(\tilde{\tau}_{i}\right)|0\rangle
$$

where $\hat{H}_{\tilde{\tau}, \mathbf{k}}^{(r)}\left(\tilde{\tau}_{i}\right)$ is the Hamiltonian operator given by

$$
\hat{H}_{\tilde{\tau}, \mathbf{k}}^{(r)}=-\frac{\hbar^{2}}{2} \frac{\partial^{2}}{\partial\left(Q_{\mathbf{k}}^{r}\right)^{2}}+\frac{1}{2} \tilde{\omega}_{k}^{2}\left(Q_{\mathbf{k}}^{r}\right)^{2}
$$

evaluated at the initial time ${ }^{7} \tilde{\tau}=\tilde{\tau}_{i}$, and $|0\rangle$ is the harmonic-oscillator ground state for the frequency $\tilde{\omega}_{k}\left(\tilde{\tau}_{i}\right)$. In a Wentzel-Kramers-Brillouin (WKB) approximation, we expand the classical test field solution $v_{k}^{r}$ as

$$
v_{k}^{r}(\tilde{\tau})=\frac{1}{\sqrt{2 \tilde{\omega}_{k}}} \exp \left[i \int d \tilde{\tau} \tilde{\omega}_{k}(\tilde{\tau})\right] .
$$

If the Hamiltonian (5.12) possesses an adiabatic regime, in which

$$
\tilde{\omega}_{k}^{\prime}=d \tilde{\omega}_{k} / d \tilde{\tau} \ll \tilde{\omega}_{k}^{2},
$$

then $v_{k}^{r^{\prime}}=d v_{k}^{r} / d \tilde{\tau}=i \tilde{\omega}_{k} v_{k}^{r}$. In the adiabatic regime where inequality (5.14) holds, the annihilation operator (5.9) (and its Hermitian conjugate $\hat{A}_{\mathrm{k}}^{\dagger}$ ) reduces to the annihilation (and creation) operator of the usual harmonic oscillator for the fixed frequency $\tilde{\omega}_{k}$ :

$$
\begin{aligned}
& \hat{A}_{\mathbf{k}}=v_{k}^{r}(\tilde{\tau})\left[\tilde{\omega}_{k} \hat{Q}_{\mathbf{k}}^{r}+\left(\partial / \partial Q_{\mathbf{k}}^{r}\right)\right], \\
& \hat{A}_{\mathbf{k}}^{\dagger}=v_{k}^{r *}(\tilde{\tau})\left[-\tilde{\omega}_{k} \hat{Q}_{\mathbf{k}}^{r}+\left(\partial / \partial Q_{\mathbf{k}}^{r}\right)\right] .
\end{aligned}
$$

Then, we can define a (usual harmonic-oscillator) number operator as

$$
\hat{N}_{k}^{r}:=\hat{A}_{\mathbf{k}}^{\dagger} \hat{A}_{\mathbf{k}}=\left|v_{k}^{r}\right|^{2}\left[\partial^{2} / \partial\left(Q_{\mathbf{k}}^{r}\right)^{2}-\tilde{\omega}_{k}^{2}\left(\hat{Q}_{\mathbf{k}}^{r}\right)^{2}\right],
$$

\footnotetext{
7 This can be considered as the initial quantum bounce at $\tilde{\tau}_{i}=\tilde{\tau}_{\mathrm{B}}$.
}

so that the wave functions (5.8) are its eigenfunctions at all times:

$$
\hat{N}_{k}^{r} \chi_{n}(Q, \tilde{\tau})=n \chi_{n}(Q, \tilde{\tau}) .
$$

This fact will lead to the interpretation of the particle number in the following.

Since $\left\{\chi_{n}\right\}$ form a complete orthonormal set for all $\tilde{\tau}$, we can expand the vacuum state $|0\rangle$ in the eigenstates $\chi_{n}$ evaluated at $\tilde{\tau}_{i}$ :

$$
|0\rangle=\sum_{n} b_{n} \chi_{n}\left(Q, \tilde{\tau}_{i}\right) .
$$

Now, we are able to calculate the expectation value of the number operator $\hat{N}_{k}^{r}$ with respect to the vacuum $|0\rangle$ as

$$
\left\langle 0\left|\hat{N}_{k}^{r}\right| 0\right\rangle=\frac{1}{2}\left(\tilde{\omega}_{i}\left|v_{k}^{r}\right|^{2}+\frac{\left|v_{k}^{r^{\prime}}\right|^{2}}{\tilde{\omega}_{i}}-1\right),
$$

where $\tilde{\omega}_{i}=\tilde{\omega}\left(\tilde{\tau}_{i}\right)$.

If the condition (5.14) is valid for all $\tilde{\tau} \geq \tilde{\tau}_{i}$, so that the system is always adiabatic, the number of particle production (5.20) for the general solution (5.13) can be expanded as

$$
\left\langle 0\left|\hat{N}_{k}^{r}\right| 0\right\rangle \approx \frac{1}{4}\left(\frac{\tilde{\omega}_{i}}{\tilde{\omega}_{k}}+\frac{\tilde{\omega}_{k}}{\tilde{\omega}_{i}}-2\right)+\mathcal{O}\left(\tilde{\omega}_{k}^{\prime} / \tilde{\omega}_{k}^{2}\right) .
$$

In the case of test-field approximation (i.e., in the absence of backreaction effects), as long as the adiabaticity condition on the background spacetime holds, the number of particle creation (5.20) reduces to ${ }^{8}$

$$
\left\langle 0\left|\hat{N}_{k}^{r}\right| 0\right\rangle=0+\mathcal{O}\left(\tilde{\omega}_{k}^{\prime} / \tilde{\omega}_{k}^{2}\right) .
$$

Thus, when the system is always adiabatic, (almost) no particles are created [32]. However, in the presence of backreaction effects (following the Born-Oppenheimer approximation), the form of the Schrödinger equation (5.4) is different from the classical one (2.27) so that we expect a modified number of particle production [up to the leading-order terms in $\sigma(k)]$ as

$$
\left\langle 0\left|\hat{N}_{k}^{r}\right| 0\right\rangle \approx \lambda^{2} \sigma^{2}+\mathcal{O}\left(\lambda^{3}\right)+\mathcal{O}\left(\tilde{\omega}_{k}^{\prime} / \tilde{\omega}_{k}^{2}\right) .
$$

Note that, for each mode, the number of created particles must be twice the result given by Eq. (5.23) because of two polarization scalar modes. So, each mode contains

$$
\left\langle 0\left|\hat{N}_{k}\right| 0\right\rangle=\sum_{r}^{2}\left\langle 0\left|\hat{N}_{k}^{r}\right| 0\right\rangle \approx 2 \lambda^{2} \sigma^{2}
$$

amount of particles in the adiabatic limit. Therefore, backreaction of the EM field on the quantum geometry

\footnotetext{
8 cf. Ref. [32] for the case in which the background is purely classical.
} 
can give rise to the particle production rate (5.24). The number of created particles depends on the energy of the field modes. So, modes with higher energies probe higher amount of particle creation. Notice that we have considered only the first-order perturbations in $\lambda$ in the mode eigenenergy, but considering higher than quadraticorder terms of $\lambda$ in perturbations might lead to the higher creation amount.

\section{CONCLUSION}

In this paper, we considered an EM field propagating on a classical flat FLRW spacetime coupled to an irrotational dust field $T$ as a background matter source. We showed that the Hamiltonian of the EM field can be written as the Hamiltonian of decoupled harmonic oscillators, each corresponding to a single mode and polarization of the field. Using the well-known procedure of loop quantum cosmology coupled to dust [10], the background geometry was quantized, in which $T$ played the role of time in quantum theory. In quantum theory, the total Hamiltonian constraint was solved in order to get a $T$ evolution for the total wave function of the system. To make a comparison between the evolution equations of the field wave function on the classical background with that given on the quantum background geometry, we employed two different techniques, namely, the test-field and the Born-Oppenheimer approximations.

By applying a test-field approximation into the total wave function of the system, we disregarded the backreaction between the field and the geometry. We obtained an effective (classical) background on which quantum modes of the EM field propagate. This emerging effective background has the same form of the original FLRW spacetime, but with the metric components depending on the quantum fluctuation of the original background geometry. Nevertheless, by taking the backreaction effects into consideration, through a Born-Oppenheimer approximation, we found an effective geometry emerging, the components of which depend on the both quantum geometry fluctuations and the modes of the field. More precisely, EM fields with different energies probe different backgrounds provided, leading to a rainbow metric forming. This result gives rise to violation of the local Lorentz symmetry on the dressed geometry.

Finally, we presented a mechanism for particle production in an adiabatic regime on the resulting effective backgrounds. Our computation showed that the amount of particle creation depends on the modifications provided by backreaction effects through Born-Oppenheimer approximation. In particular, when backreaction was discarded, no amount of particles could be detected. However, when the backreaction was taken into account, EM field modes could probe particle creations. Moreover, the number of particle production depended on the mode of the field; that is, modes with higher energy detected a higher amount of particle production and vice versa.

\section{Acknowledgments}

The authors would like to thank N. Ahmadi for the useful comments on quantum theory of EM field in a classical background. M. N-Z. and A. P. thank the research council of the University of Tehran for financial support. Y. T. acknowledges Bonyad-e-Melli Nokhbegan of Iran (INEF) for financial support, and the Brazilian agencies FAPES and CAPES for partial financial support. He thanks Chun-Yen Lin, T. Pawlowski, and S. Sheikh-Jabbari for very useful discussions on this work. He is also grateful for the warm hospitality of the University of Warsaw where part of this work was completed. This work was supported by the grant of Polish Narodowe Centrum Nauki, Grant No. 2011/02/A/ST2/00300.

\section{Appendix A: Connection coefficients and Ricci tensor in the FLRW spacetime}

For the metric (2.5), the nonzero connection coefficients and Ricci tensor in the rectangular coordinates are given by the following expressions:

$$
\begin{aligned}
& \Gamma_{00}^{0}=\frac{\dot{N}}{N}, \quad \Gamma_{x x}^{0}=\Gamma_{y y}^{0}=\Gamma_{z z}^{0}=\frac{a \dot{a}}{N^{2}}, \\
& \Gamma_{0 x}^{x}=\Gamma_{0 y}^{y}=\Gamma_{0 z}^{z}=\frac{\dot{a}}{a},
\end{aligned}
$$

and

$$
\begin{aligned}
& \mathcal{R}_{00}=\frac{3 \ddot{a} N-\dot{N} \dot{a}}{a N}, \\
& \mathcal{R}_{x x}=\mathcal{R}_{y y}=\mathcal{R}_{z z}=-\frac{a \ddot{a} N-a \dot{N} \dot{a}+2 N \dot{a}^{2}}{N^{3}} .
\end{aligned}
$$

These connection coefficients indicate that there are no terms involving first-order spatial derivatives of the components of EM potential in the wave equation (2.4).

\section{Appendix B: Hamiltonian of the EM field in the FLRW spacetime}

We can rewrite the Lagrangian density (2.1) as

$$
\mathcal{L}_{\mathrm{EM}}=-\frac{1}{2} \sqrt{-g}\left[\sum_{i}^{3} F_{0 i} F^{0 i}+\sum_{i<j}^{3} F_{i j} F^{i j}\right] .
$$

By fixing the gauge condition $A^{0}=0$, for which $F_{0 i}=$ $\partial_{0} A_{i}$ and $F^{0 i}=\partial^{0} A^{i}$, Eq. (B1) reduces to

$$
\begin{gathered}
\mathcal{L}_{\mathrm{EM}}=-\frac{1}{2} \sqrt{-g}\left[\sum_{i} \sum_{j}\left(\partial_{0} A_{i}\right) g^{00} g^{i j}\left(\partial_{0} A_{j}\right)\right. \\
\left.+\sum_{i<j} F_{i j} F^{i j}\right] .
\end{gathered}
$$


The conjugate momentum $\pi^{i}$ for the vector fields $A_{i}$ can be defined as

$$
\begin{aligned}
\pi^{0} & =\frac{\delta \mathcal{L}_{\mathrm{EM}}}{\delta\left(\partial_{0} A_{0}\right)}=0, \\
\pi^{i} & =\frac{\delta \mathcal{L}_{\mathrm{EM}}}{\delta\left(\partial_{0} A_{i}\right)}=-\sqrt{-g} F^{0 i}=E^{i} .
\end{aligned}
$$

Notice that $\pi^{i}$ is conjugate to $A_{i}=g_{i j} A^{j}=a^{2} A^{i}$. Then, the usual commutation relations read

$$
\left[A_{i}\left(x_{0}, \mathbf{x}\right), \pi^{j}\left(x_{0}, \mathbf{x}^{\prime}\right)\right]=i \delta^{i}{ }_{j}
$$

By using $\mathcal{H}_{\mathrm{EM}}=\pi^{i}\left(\partial_{0} A_{i}\right)-\mathcal{L}_{\mathrm{EM}}$, the Hamiltonian density is written as

$$
\mathcal{H}_{\mathrm{EM}}=\frac{1}{2} \sqrt{-g}\left[\sum_{i} \frac{g_{00}}{g}\left(\pi_{i} \pi^{i}\right)+\sum_{i<j} F_{i j} F^{i j}\right] .
$$

For the flat FLRW background (2.5), the Hamiltonian density of EM field (B6) reduces to

$$
\mathcal{H}_{\mathrm{EM}}=\frac{N_{x_{0}}}{2 a^{3}}\left[\sum_{i} \pi_{i} \pi^{i}+a^{6} \sum_{i<j} F_{i j} F^{i j}\right] .
$$

To calculate the last term in the Hamiltonian density (B7), we write

$$
\begin{aligned}
\sum_{i<j} F_{i j} F^{i j} & =F_{12} F^{12}+F_{13} F^{13}+F_{23} F^{23} \\
& =\sum_{i, j}^{3}\left[\left(\partial_{i} A_{j}\right)\left(\partial^{i} A^{j}\right)-\left(\partial_{i} A_{j}\right)\left(\partial^{j} A^{i}\right)\right] .
\end{aligned}
$$

We define the covariant derivative in three-dimensional space in the same way we define the four-dimensional covariant derivative; then, $A_{a \mid b}$ shows the threedimensional covariant derivative in terms of partial derivatives and connections of the given 3 -space $(\Sigma, \gamma)$,

$$
\nabla_{j} A^{i}:=A_{\mid j}^{i}=\partial_{j} A^{i}+r^{i}{ }_{j k} A^{k},
$$

where $r_{j k}^{i}$ are connections of the 3-space, and the 3dimensional divergence of $\mathbf{A}$ reads

$$
\nabla \cdot \mathbf{A}=A_{\mid i}^{i}=\frac{1}{\sqrt{\gamma}} \frac{\partial}{\partial_{i}}\left(\sqrt{\gamma} A^{i}\right) .
$$

In terms of the three-dimensional covariant derivative, Eq. (B8) takes the following form:

$$
\begin{aligned}
F_{i j} F^{i j} & =2\left[\left(\partial_{i} A_{j}\right)\left(\partial^{i} A^{j}\right)-\left(\partial_{i} A_{j}\right)\left(\partial^{j} A^{i}\right)\right] \\
& =2\left[\nabla_{i} A_{j} \nabla^{i} A^{j}-\nabla_{i} A_{j} \nabla^{j} A^{i}\right] .
\end{aligned}
$$

The second term in the equation above can be written as a total divergence:

$$
\left(\nabla_{i} A_{j}\right)\left(\nabla^{j} A^{i}\right)=\nabla_{i}\left(A_{j} \nabla^{j} A^{i}\right)-A_{j}\left(\nabla_{i} \nabla^{j} A^{i}\right) .
$$

In the summation, the last term in the equation above reads $A_{j}\left(\nabla^{j} \nabla_{i} A^{i}\right)=A_{j} \nabla^{j}(\nabla \cdot \mathbf{A})$. Then, in the radiation gauge, $\nabla \cdot \mathbf{A}=0$, the last term vanishes. Thus, the total divergence is equal to the first term only, which vanishes by integration with respect to the spatial volume when computing the total Hamiltonian $H_{\mathrm{EM}}$, so that only the first term in Eq. (B8) remains nonzero. On the other hand, for the first term, we can also write

$$
\left(\nabla_{i} A_{j}\right)\left(\nabla^{i} A^{j}\right)=\nabla_{i}\left(A_{j} \nabla^{i} A^{j}\right)-A_{j} \nabla_{i} \nabla^{i} A^{j} .
$$

Using the divergence theorem in three-dimensional curved space, we have that

$$
\int_{\Sigma} \sqrt{\gamma} d x^{3} A^{i}{ }_{i i}=\oint_{\partial \Sigma} A^{i} d \Sigma_{i}
$$

where $\gamma=a^{6}$ herein our background spacetime. Then, we obtain

$$
\int \sqrt{\gamma} d x^{3}\left(\nabla_{i} A_{j}\right)\left(\nabla^{i} A^{j}\right)=-\int \sqrt{\gamma} d x^{3} A_{j} \nabla^{2} A^{j} .
$$

Using the ingredients provided in the paragraph above, by integrating over the Hamiltonian density (B7), we obtain the total Hamiltonian (2.16) for the EM field. The total Hamiltonian (2.16) can be split through positive and negative sectors on the lattice $\mathcal{L}$ as

$$
\begin{aligned}
& H_{\mathrm{EM}}= \sum_{\mathbf{k} \in \mathcal{L}_{+}} \sum_{r} H_{\mathbf{k}}^{(r)+}+\sum_{\mathbf{k} \in \mathcal{L}_{-}} \sum_{r} H_{\mathbf{k}}^{(r)-} \\
&= \frac{N_{x_{0}}}{2 a^{3}} \sum_{r}\left[\sum_{\mathbf{k} \in \mathcal{L}_{+}}\left(\left(\pi_{\mathbf{k}}^{r}\right)^{*} \pi_{\mathbf{k}}^{r}+k^{2} a^{4}\left(\mathrm{~A}_{\mathbf{k}}^{r}\right)^{*} \mathrm{~A}_{\mathbf{k}}^{r}\right)\right. \\
&\left.\quad+\sum_{\mathbf{k} \in \mathcal{L}_{-}}\left(\left(\pi_{\mathbf{k}}^{r}\right)^{*} \pi_{\mathbf{k}}^{r}+k^{2} a^{4}\left(\mathrm{~A}_{\mathbf{k}}^{r}\right)^{*} \mathrm{~A}_{\mathbf{k}}^{r}\right)\right] \\
&= \frac{N_{x_{0}}}{2 a^{3}} \sum_{r} \sum_{\mathbf{k} \in \mathcal{L}_{+}}\left[\left(\pi_{\mathbf{k}}^{r}\right)^{*} \pi_{\mathbf{k}}^{r}+\left(\pi_{-\mathbf{k}}^{r}\right)^{*} \pi_{-\mathbf{k}}^{r}\right. \\
&\left.\quad+k^{2} a^{4}\left(\left(\mathrm{~A}_{\mathbf{k}}^{r}\right)^{*} \mathrm{~A}_{\mathbf{k}}^{r}+\left(\mathrm{A}_{-\mathbf{k}}^{r}\right)^{*} \mathrm{~A}_{-\mathbf{k}}^{r}\right)\right] \cdot(\mathrm{B} 12)
\end{aligned}
$$

In the last step above, by a simple changing of index $\mathbf{k} \rightarrow-\mathbf{k}$, we have converted the sum over the negative index to that over the positive index. Substituting now $\mathrm{A}_{\mathbf{k}}^{r}$ and $\pi_{\mathbf{k}}^{r}$ from Eqs. (2.17) and (2.18) into Eq. (B12), we obtain

$$
\begin{aligned}
H_{\mathrm{EM}}= & \frac{N_{x_{0}}}{4 a^{3}} \sum_{\mathbf{k} \in \mathcal{L}_{+}} \sum_{r} \sum_{n=1}^{2}\left[\left(\pi_{\mathbf{k}}^{r(n)}\right)^{2}+\left(\pi_{-\mathbf{k}}^{r(n)}\right)^{2}\right. \\
& \left.+k^{2} a^{4}\left(\left(\mathrm{~A}_{\mathbf{k}}^{r(n)}\right)^{2}+\left(\mathrm{A}_{-\mathbf{k}}^{r(n)}\right)^{2}\right)\right] \\
= & \frac{N_{x_{0}}}{2 a^{3}} \sum_{\mathbf{k} \in \mathcal{L}_{+}} \sum_{r} \sum_{n=1}^{2}\left[\left(\pi_{\mathbf{k}}^{r(n)}\right)^{2}+k^{2} a^{4}\left(\mathrm{~A}_{\mathbf{k}}^{r(n)}\right)^{2}\right] .
\end{aligned}
$$

This equation represents a Hamiltonian for a collection of independent harmonic oscillators. We can further rewrite 
the last relation in Eq. (B13) as sum of modes of two harmonic oscillators:

$$
\begin{aligned}
& H_{\mathrm{EM}}= \frac{N_{x_{0}}}{2 a^{3}} \sum_{\mathbf{k} \in \mathcal{L}_{+}} \sum_{r}\left\{\left[\left(\pi_{\mathbf{k}}^{r(1)}\right)^{2}+k^{2} a^{4}\left(\mathrm{~A}_{\mathbf{k}}^{r(1)}\right)\right)^{2}\right] \\
&\left.\left.+\left[\left(\pi_{\mathbf{k}}^{r(2)}\right)^{2}+k^{2} a^{4}\left(\mathrm{~A}_{\mathbf{k}}^{r(2)}\right)\right)^{2}\right]\right\} \\
&=\frac{N_{x_{0}}}{2 a^{3}} \sum_{\mathbf{k} \in \mathcal{L}_{+}} \sum_{r}\left[\left(\pi_{\mathbf{k}}^{r(1)}\right)^{2}+k^{2} a^{4}\left(\mathrm{~A}_{\mathbf{k}}^{r(1)}\right)^{2}\right] \\
&+\frac{N_{x_{0}}}{2 a^{3}} \sum_{\mathbf{k} \in \mathcal{L}_{-}} \sum_{r}\left[\left(\pi_{-\mathbf{k}}^{r(2)}\right)^{2}+k^{2} a^{4}\left(\mathrm{~A}_{-\mathbf{k}}^{r(2)}\right)^{2}\right] .
\end{aligned}
$$

Now, by defining the new real variables (2.22) and (2.23) and substituting in Eq. (B14), the total Hamiltonian (2.24) is obtained.
[1] A. Ashtekar and J. Lewandowski, Background independent quantum gravity: A Status report, Classical Quantum Gravity 21, R53 (2004).

[2] C. Rovelli, Quantum Gravity (Cambridge University Press, Cambridge, England, 2004).

[3] T. Thiemann, Introduction to Modern Canonical Quantum General Relativity, (Cambridge University Press, Cambridge, England, 2007).

[4] A. Ashtekar, W. Kaminski and J. Lewandowski, Quantum field theory on a cosmological, quantum spacetime, Phys. Rev. D 79, 064030 (2009).

[5] A. Dapor, J. Lewandowski, and Y. Tavakoli, Lorentz symmetry in QFT on quantum Bianchi I spacetime, Phys. Rev. D 86, 064013 (2012).

[6] A. Dapor, J. Lewandowski, and J. Puchta, QFT on quantum spacetime: a compatible classical framework, Phys. Rev. D 87, 104038 (2013).

[7] A. Ashtekar, M. Bojowald, and J. Lewandowski, Mathematical structure of loop quantum cosmology, Adv. Theor. Math. Phys. 7, 233 (2003).

[8] A. Ashtekar and P. Singh, Loop Quantum Cosmology: A Status Report, Classical Quantum Gravity 28, 213001 (2011).

[9] A. Ashtekar, T. Pawlowski, and P. Singh, Quantum Nature of the Big Bang, Phys. Rev. Lett. 96, 141301 (2006).

[10] V. Husain and T. Pawlowski, Time and a physical Hamiltonian for quantum gravity, Phys. Rev. Lett. 108, 141301 (2012).

[11] A. Dapor and J. Lewandowski, Metric emerging to massive modes in quantum cosmological spacetimes, Phys. Rev. D 87, 063512 (2013).

[12] M. Assanioussi and A. Dapor, Rainbow metric from quantum gravity: anisotropic cosmology, arXiv:1606.09186 [gr-qc].

[13] M. Assanioussi, A. Dapor, and J. Lewandowski, Rainbow metric from quantum gravity, Phys. Lett. B 751, 302(2015).

[14] I. Agullo, A. Ashtekar, and W. Nelson, A Quantum Gravity Extension of the Inflationary Scenario, Phys. Rev. Lett. 109, 251301 (2012).

[15] G. Amelino-Camelia, M. Arzano, G. Gubitosi, and J. Magueijo, Dimensional reduction in the sky, Phys. Rev. D 87, 123532 (2013).

[16] G. Amelino-Camelia, J. Ellis, N. E. Mavromatos, D. V. Nanopoulos, and S. Sarkar, Tests of quantum gravity from observations of $\gamma$-ray bursts, Nature (London) 393, 763 (1998).
[17] D. Mattingly, Modern tests of Lorentz invariance, Living Rev. Relativity 8, 5 (2005).

[18] L. Smolin, Falsifiable predictions from semiclassical quantum gravity, Nucl. Phys. B742, 142 (2006).

[19] G. Amelino-Camelia, L. Freidel, J. Kowalski-Glikman, and L. Smolin, The principle of relative locality, Phys. Rev. D 84, 084010 (2011).

[20] J. Magueijo, Speedy sound and cosmic structure, Phys. Rev. Lett. 100, 231302 (2008).

[21] R. Lafrance and R. C. Myers, Gravity's rainbow: Limits for the applicability of the equivalence principle, Phys. Rev. D 51, 2584 (1995).

[22] J. Magueijo and L. Smolin, Gravity's rainbow, Classical Quantum Gravity 21, 1725 (2004).

[23] T. Shinji, P. Singh, and R. Maartens, Loop quantum gravity effects on inflation and the CMB, Classical Quantum Gravity 21, 5767 (2004).

[24] C. W. Misner, K. S. Thorne, and J. Archibald Wheeler, Gravitation (Freeman, San Francisco, 1973).

[25] Lewis H. Ryder, Quantum Field Theory (Cambridge University Press, Cambridge, England, 1985).

[26] V. Mukhanov and S. Winitzki, Introduction to Quantum Effects in Gravity (Cambridge University Press, Cambridge, England, 2007)

[27] W. Kaminski, J. Lewandowski, and T. Pawlowski, Quantum constraints, Dirac observables and evolution: group averaging versus the Schrdinger picture in LQC, Classical Quantum Gravity 26, 245016 (2009).

[28] C. Kiefer, Quantum Gravity (Oxford University, New York, 2004).

[29] C. Rovelli and F. Vidotto, Stepping out of Homogeneity in Loop Quantum Cosmology, Classical Quantum Gravity 25, 225024 (2008).

[30] K. Giesel, J. Tambornino, and T. Thiemann, BornOppenheimer decomposition for quantum fields on quantum spacetimes, arXiv:0911.5331.

[31] Y. Tavakoli and J. C. Fabris, Creation of particles in a cyclic universe driven by loop quantum cosmology, Int. J. Mod. Phys. D 24, 1550062 (2015).

[32] B. K. Berger, Scalar particle creation in an anisotropic universe, Phys. Rev. D 12, 368 (1975).

[33] A. M. Perelmov and V. S. Popov, Parametric Excitation of a Quantum Oscillator, Zh. Eksp. Fiz. 56, 1375 (1969) [Sov. Phys. JETP 29, 738 (1969)].

[34] E. Salusti and F. Zirilli, On the time-dependent harmonic oscillators, Lett. Nuovo Cimento Lett. 4, 999 (1970).

[35] Ya. B. Zel'dovich, Particle Production in Cosmology, 
Pisma Zh. Eksp. Teor. Fiz. Pis'ma Red. 12, 443 (1970)

[JETP Lett. 12, 307 (1971)]. 